\title{
A study of the iPhone app at Kew Gardens: Improving the visitor experience
}

\author{
Caryl Mann \\ The Susie Fisher Group \\ 47 Hardel Walk, London, SW2 2QG \\ carylharp@gmail.com
}

\begin{abstract}
In July 2011 Kew Gardens launched an app called 'Kew' with the aim of enabling visitors to "explore more of Kew gardens and dig deeper into [the] science and horticulture" (Kew Royal Botanic Gardens 2012). The Kew app is designed to facilitate this experience by providing location specific information on a customizable map through seasonal recommendations, and site specific tools, such as QR code reader for plant labels and an augmented reality browser, enabling a more personalized and unique exploration of Kew. In October 2011 audience research was carried out to evaluate visitors responses and experiences to the app and to identify the nature of their experience of Kew when using the app.

This paper will explore the viewpoint of the visitors using the app and show that a seamless experience was an important factor in visitor satisfaction. What impact do technological successes and problems have on a seamless experience and how do visitor expectations define seamlessness? To illustrate the importance of seamlessness specific functions of the app are outlined in terms of user observation and experience of this principle. This will provide insights and guidelines for the use of mobile technology in other museums.
\end{abstract}

Mobile, apps, audience research, seamlessness, evaluation, visitor

\section{INTRODUCTION}

Mobile technologies are becoming an increasingly integral part of the visitor experience in museums and heritage sites. With the focus on creating a visitor-centred and interactive experience in exhibitions, digital technologies offer the potential to enhance the visitor experience in exciting new ways. This paper explores the visitor experience of an iPhone app at Kew Gardens more clearly by reporting observations of visitors during fieldwork for a qualitative study of visitor experiences of the app. A striking observation was the correlation between visitor expectation of, and familiarity with, the technology, motivations for visiting, and familiarity with Kew, and how these determined visitor flow around the Gardens. It will be shown that an important component in the visitor experience of mobile technology is Mark Weiser's concept of seamlessness where technology becomes invisible and recedes into the background:

The most potentially interesting, challenging, and profound change implied by the ubiquitous computing era is a focus on calm. "If computers are everywhere they had better stay out of the way, and that means designing them so that the people being shared by the computers remain serene and in control ... [c]omputers for personal use have focused on the excitement of interaction. But when computers are all around, so that we want to compute while doing something else and have more time to be more fully human, we must radically rethink the goals, context and technology of the computer and all the other technology crowding into our lives" (Weiser and Brown 1996). 
Table 1: Audience Segmentation

\begin{tabular}{|l|l|l|}
\hline Target audience & Audience segment & Needs during visit \\
\hline Primary & Learning families & $\begin{array}{l}\text { Child friendly facilities and activities, } \\
\text { fun activities, }\end{array}$ \\
\hline & Leisure & $\begin{array}{l}\text { Peace, unobtrusive signage, succinct } \\
\text { interpretation }\end{array}$ \\
\hline Secondary & Site seers (social first timers) & $\begin{array}{l}\text { Orientation, clear signage, } \\
\text { Engaging tours }\end{array}$ \\
\hline & Social spacers (older repeat visitors) & Orientation, fun and engaging tours \\
\hline
\end{tabular}

In this vision of ubiquitous computing, systems such as GPS, Wi-Fi, 3G, and digital cameras work together seamlessly so that technology recedes into the background or periphery of the user's awareness. The goal of a seamless integration of computer and people posits that design must always be for context: design for the periphery so technology does not dominate in the centre. In Kew when the app is on the periphery visitors are focused on the central task of exploring the Garden. Also important is the notion that what is on the periphery can move to the centre and back again, the app switching to central focus as visitors look at image content, returning to the periphery again as the images of plants are discovered (Weiser and Brown 1996).

\section{THE KEW IPHONE APP: DELIGHTFULLY LOST}

The Royal Botanic Gardens at Kew is a leading international scientific research institution and world heritage site that attracted 1, 188, 933 visitors in 2011 to view the collection of diverse varieties of plants in grounds that encompass 300 acres of land (Kew Royal Botanic Gardens). Although it would be expected that orientation would be a key concern of visitors to the Gardens, to paraphrase Natasha Waterson, Senior Producer of Mobile Services at Kew, audience research carried out in October 2010 had revealed that visitors to Kew were primarily motivated by social, emotional and spiritual reasons for their visit, as opposed to intellectual, and enjoyed a meandering exploration or serendipitous discovery of the gardens (Saunders and Waterson 2012).

This understanding of audience motivations informed the development of the Kew app and "delightfully lost" was adopted as a guiding metaphor for a design that would fit into this purpose. The app would not provide a structure or route but provide triggers or suggestions of things to explore, taking visitors in new directions and away from habitual routes. The visitor would not be wedded to the phone visually but the app would be on the periphery - moving to the centre -back to the periphery as little or often as the visitor required
(Saunders and Waterson 2012). Therefore, to facilitate a serendipitous discovery of the Gardens the app includes the following features: A map with customization options according to visitor interests, with GPS location detection technology for orientation; augmented reality to prompt discovery and location of trees and encourage meandering exploration; a QR code reader so visitors could scan plants and link to in-app content with detailed text or audio-visual information; See today at Kew which contains images from the Flickr social image sharing website uploaded by Kew and visitors, again tempt investigation and pull visitors away from their habitual routes; a Gallery of images grouped in themes for suggested themes for walking around the Gardens. Together these features present a culture iPhone app that is both a new medium in which to deliver conventional information and, through digital technologies such as augmented reality, a new concept in the visitor experience to Kew Gardens.

\section{AUDIENCE RESEARCH: METHODS AND TECHNIQUES}

In October 2012 the Susie Fisher Group was commissioned to research the visitor experience of the Kew iPhone app during visits to the gardens. This was a qualitative research study focused on key target audiences and current high iPhone users (Susie Fisher Group 2011). The audience groups selected from the motivational segmentation for the study included leisure and learning families, sightseers who were first timers to the Gardens, and social spacers, also repeat visitors (Table 1) (Saunders and Waterson 2012).

The methodology for the research was a combination of face-to-face qualitative interviewing and observation of visitor behaviour during accompanied visits. The surveyed visitors were iPhone users, had downloaded the app and already used it in the Gardens (Saunders and Waterson 2012). Age was not a defining factor as the focus was on motivation for visiting and familiarity with the iPhone, so each group included a broad age-range. The evaluation was with: 
(i) Two focus groups of eight participants from two audience groups: families who were regular visitors to Kew visiting with children under fifteen, and sightseers who had visited Kew once or twice for social reasons (four men and four women, aged 26-60).

(ii) Six accompanied visits with interviews, with participants from each audience group: family, sightseers, leisure and social spacers (three men and four women, ages 25-45, a mix of regular and infrequent users) (Saunders and Waterson 2012).

The goals of the evaluation were two-fold: to understand how the app integrated into existing modes of visiting; and, to identify whether the app changed the nature of the visitor experience, thereby providing a new concept or type of experience. The research had specific evaluation questions but rather than focus on these I will look at each feature of the app in relation to a seamless experience.

\section{THE VISITOR EXPERIENCE}

\subsection{See today at Kew}

Visitors from each segment responded positively to this feature and felt it enhanced their visit to Kew. When visitors click on See Today they are connected to highlights and alerts, with images, uploaded onto Flickr by Kew experts recommending what to see in the Gardens. In Museums and their visitors Hooper-Greenhill (1994) identifies an iconic mode of learning that consists of images and representations of reality that provides a concrete experience. This was evident in visitor responses to See Today as the images operated as a powerful prompt to discover the real-life plant: all visitors incorporated this part of the app into their visit. This was true both of visitors who wished to include the recommendation in their planned route and those who wished simply to meander spontaneously.

The pattern of visits consisted of: look at See Today $>$ identify the location of the attraction $>$ go in search of it $>$ find the attraction $>$ expectation fulfilled. The app worked seamlessly, moving from the centre to the periphery, as the plant became the focus of the visit and created an enhanced visit that felt unique to the app:

Hoping to see something we haven't seen before....because it's a massive place...I think See Today has the potential [to show more of Kew] for you...See Today is a really good feature (Social spacer).
Look at what's on today. Do this bit for a couple of hours...it encourages you to do stuff, to prepare your visit and go to new places (Mum).

Some visitors experienced problems finding the location of the attraction and this disrupted the seamless experience. A female sightseer in her twenties using See Today went in search of the papayas. This exploration was thwarted however, because "the papaya arrow wasn't exact...I never found the papayas." Here the technology became visible and frustrated the visit, pointing towards a lack of seamlessness between the GPS and See Today.

\subsection{The map and GPS}

The map and GPS were welcomed by visitors and seen as a core function of the app. Like See Today the map had been designed to function equally as part of a meandering, serendipitous visit, or as part of a planned itinerary depending on visitor motivation. Both features were vital for locating plants in See Today, whilst GPS was vital for locating trees for augmented reality. Consequently, both features were an important part of the visitor experience with the iPhone at Kew.

When opening the map visitors see a comprehensive map of Kew, but without all attractions labeled. The See Today highlights appear as 'eye' icons (see Figure 2) that visitors can tap to reveal the location and connect to a Flickr website.

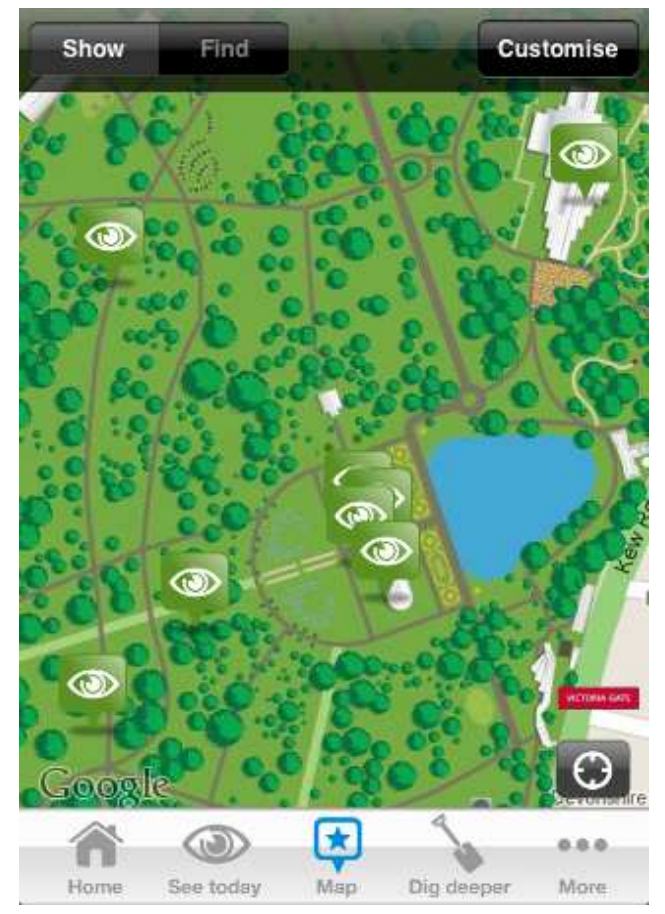

Figure 2 Kew iPhone app: Map 
Visitors also have the option to search for plants or attractions in the 'Search' button and locate the interest on the map, and customize the map and add information, such as Views or even toilets and cafes, by clicking on the 'Customise' button. Lastly, the map has GPS functionality and visitors can locate themselves on the map and navigate their way around the Gardens.

The map provides a considerable degree of freedom for the visitor to personalize their visit and create their own itinerary, breaking away from their habitual routes to discover more of Kew. The option to tap on an icon and instantly receive deeper information was found to contribute to the experience of a spontaneous visit and offer learning opportunities. When successful the visitor experience was one of "delightfully lost". Some positive results of the map included:

I love the source of information there. Tap on
the icon, where you are (Mum).

You can start at a different gate. Come in at a different gate and rediscover a grove. It's beautiful (Mum).

You can bring up a picture and find it on the map and go there (Sightseer).

I wanted to fund the Princess Diana Conservatory. I typed in Princess Diana and it showed up. And I looked up the Palm House on the app (Sightseer).

Vintage views altered [my] experience of Kew as any history interested in, so just for that alone would come back to Kew (Leisure visitor).

The map was not a seamless experience for visitors who came with different expectations of and familiarity with iPhone maps as a result of being disciplined by the Google Maps app. The widespread use of Google Maps conditions user perceptions of what is intuitive or logical interface for a map. For example, a map is presented with a dense amount of data that appears visually cluttered. This informs the user to customise and remove information. Consequently, the uncluttered appearance of the Kew app map communicated to some visitors that customization was not an option. This was further compounded by a customise icon that went unnoticed because it differed from the Google Maps icon visitors had become accustomed to using. This has been updated to 'Customise' following this visitor study (see Figure 2). Finally, the GPS was not sensitive enough to provide the spatial accuracy required for successful location of plants and attractions, the app and visitor wanting and needing more than GPS technology currently allows. The results, therefore, were less successful and the map interfered with a seamless visit, whether planned or meandering.
Less successful experiences of the map included the following, the problems in some having been addressed following this qualitative study:

The map moved but the blue light wasn't there. The signal was weak. You have to stop what you're doing and wait (Sightseer).

This has been identified as a bug rather than design flaw that Kew is currently working to resolve.

It doesn't activate a compass which shows you which way you are walking. It makes me less likely to use the map. It makes me resort to the old ways of using the sign (Social spacer).

This functional improvement has now been implemented.

The map doesn't have enough icons. Because when you click on that there it doesn't tell you what it is, it just brings up plants. Not really enough attractions. There's a whole area of Kew there with no attractions according to the app (Social spacer).

Some areas of Kew, such as the Conservation Area and Arboretum, do not contain many attractions and are more of a wild space; how the app can clearly communicate this is an outstanding question.

I think you just fall into a pattern, especially with maps with that you just assume how they work, like with Google Maps (Social spacer).

It's really about information, so personalizing the map, although it would be good, [it requires] playing around with it when you've got time (Sightseer).

These results reveal a correlation between visitor motivations, familiarity with the iPhone and familiarity with Kew. For sightseers and social spacers familiar with iPhones but not Kew, the map was not intuitive and required an investment of time that interfered with their visit (Susie Fisher Group 2011: 2). The iPhone would become visible and a focus of the visit rather than invisible and seamless. For leisure or learning visitors, in particular families, already familiar with Kew, the iPhone was successful and unique to the visit at Kew.

\subsection{Augmented Reality}

This feature was seen as an exciting addition to the experience of the Gardens. The concept behind augmented reality is one of augmenting the physical environment by using GPS location technology and graphics (Wikipedia: Augmented reality). In the Kew app augmented reality is a combination of GPS, camera and compass. To use augmented reality visitors hold up the iPhone, 
and green circles appear with the names of trees. This triggers a search for a tree whilst the visitor interacts with the iPhone like a compass until the tree is discovered.

Like See Today the augmented reality worked as a powerful incentive for visitors to explore the Gardens spontaneously:

\begin{abstract}
Augmented reality. Lot of dots came up. If you moved the phone around you could identify each dot for each plant. It was really lovely. If it's interesting when I wander, it heightens my interest. (Sightseer).
\end{abstract}

Like the map visitors' experienced navigational problems with the augmented reality but this frustration was minimized by visitor enthusiasm: "The augmented reality, you hold it up and scan it. And the scan didn't come up for that tree. It showed things at the other end of the Palm House. It was confusing but it's a good idea." (Sightseer). The dots appeared inside the house because the augmented reality tree profiles were so close. There are now augmented reality profiles inside the house. However, this experience highlights the importance of an accurate search distance for visitors.

The augmented reality went unnoticed however by visitors unfamiliar with the terminology and technology:

\section{It sounds good but I just wonder what proportion of the population [would know about it]...your seventeen year old would understand, but older people would not understand augmented reality (Sightseer).}

Presently therefore, augmented reality has the potential to attract younger audiences already accustomed to interactive computing. It also creates a museum experience specific to the technology and iPhone. As this technology becomes more ubiquitous visitors may increasingly expect the type of interactive and enhanced experience provided by augmented reality.

\subsection{QR code reader}

At present QR codes have been implemented as a trial to evaluate visitor response and are limited to the Princess of Wales Conservatory, Rock Garden and eighty trees across the Gardens (Saunders and Waterson 2012). A QR or quick response code is a two-dimensional barcode that connects to web based content (Wikipedia: QR Code). Due to its fast readability and large storage capacity the QR code provides an alternative to the traditional label and can offer a greater range and depth of content. The Kew app uses accessible and informative language for the visitor to understand the technology.

However, although QR codes are becoming increasingly ubiquitous, appearing on consumer goods and in magazines, these have not become part of general computer literacy. Consequently, the QR codes were not instantly identifiable or understood by some visitors. Their responses to the QR codes included: It was much more than I thought it would
be...the video [of the plant] really impressed me.
Was expecting it to just give a history or
description and wasn't expecting a short film or
audio (Sightseer).

I'd rather read it on paper than see it as audio. I like simple boards that say, "Did you know? (Leisure visitor).

There was lots of copy and I [didn't] want to read that. It would be better with bullet points and a quick summary (Sightseer).

I really like the idea of it but couldn't get it to work (Independent visitor).

I only came across one of the QR codes which worked, which was great (Sightseer).

Like augmented reality, familiarity with the technology influenced visitor expectations that the QR codes would be ubiquitous across the Gardens: "I do find the information a bit dry but [today] I couldn't find any...haven't seen any yet" (Social spacer). These visitor responses reveal five points about QR codes in museums. Firstly, without knowledge of $\mathrm{QR}$ code visitors are unaware that scanning the barcode connects them to larger web based content, and, are therefore likely to overlook the potential of the technology. Secondly, the use of audio, visual or text content appeals to multiple learning styles and QR code technology provides a platform to meet a diverse range of audience needs. Third, these multiple learning styles need to be catered for consistently perhaps with an option for the visitor to select their preferred mode, for example audio-visual, bite-size facts or specialized interpretation (Susie Fisher Group 2011: 2). Fourth, advertising the QR codes clearly so visitors know to find these in a specific section or spread across selected objects reduce the possibility of visitors focusing attention on finding the codes and detracting attention from the experience of looking and discovering. Finally, when the QR codes do not work with a single click visitors will give up and once again the technology becomes visible and a focus of the visit rather than work seamlessly in the background (Susie Fisher Group 2011: 2).

\subsection{Wi-Fi and 3G}


The Kew app is accessed through Wi-Fi and 3G areas. During the fieldwork these connections were patchy and this resulted in delays for visitors (Susie Fisher Group 2011: 2):

I expected the app to be all there when I arrived rather than having to update it. I was waiting around for it to happen so stopped it. Not interested enough in the update to wait (Sightseer).

Signs are static but the app was also static because of the slow Wi-Fi (Leisure).

The expectation that visitors can instantly download content and connect to a network is an important part of the experience of ubiquitous computing and where this lacking visitors quickly are reluctant to wait and typically return to "the old ways of using maps and signs" (Social spacer).

\section{6 Flickr}

The Flickr page contains images uploaded by visitors and Kew experts. The idea of a 'Kew Community' on Flickr was popular amongst younger visitors already conversant with social networking and image sharing sites such as Flickr (Susie Fisher Group 2011: 2):

\begin{abstract}
It's a shared experience, you're involved in a community. You share with other people if you take your own photos. You can see other people's photos and you're wanting to go and see" (Sightseer)
\end{abstract}

Older visitors unfamiliar with Flickr did not see this feature as a tool to enhance their visit (Susie Fisher Group 2011: 2). Some concern was raised that the app did not work seamlessly from Flickr and the other features: "I clicked on an icon for more information and where to go and it went back to the photo, the Flickr page, instead" (Sightseer) (Susie Fisher Group 2011). However, following this visitor study the navigation between features has been made clearer. The main strength of the Flickr feature was a sense of sharing and dialogue between other visitors and Kew experts and the way visitors could incorporate these suggestions into their visits. The Kew app provides a potential seamless experience of Kew on and off site and possibly as a stimulus for visits.

\section{CONCLUSION}

The visitor experience of the iPhone app at Kew was mainly positive: it improved the visitor experience by deepening and expanding knowledge of the Gardens. The Kew app was also welcomed as a new addition to the Kew experience as a means of delivering old information through a new medium, and as a new companion that integrated into the mode of their visit. This positive embrace of new mobile technology in a heritage site demonstrates the extent to which ubiquitous computing is now a common and expected part of peoples' daily patterns of interaction and modes of behaviour. This provides further evidence to museums that mobile technology is a powerful tool, as both medium and concept, an encouragement to continue to make the transition from old museums visitor models of passive, didactic learning, to the new museum visitor model of interactive, collaborative and dialogic learning.

However, it is imperative that the technology works seamlessly and without disruptions that distract the visitor from the object on display, and undermine the visitor's desired museum experience. The expectation the visitor brings in terms of their motivation and mode of visit (identified in audience research and segmentation) now intersects with the expectation they bring to the museum iPhone app, or other mobile platform, and this cannot be ignored. This expectation is disciplined and shaped by existing interface design, different degrees of technological fluency and the assumption of a networked world where information can be shared easily and instantly. This determines the experience of seamlessness that underpins the principle of ubiquitous computing. This in turn determines the visitor's expectation of a museum app designed to be an inherent part of an exhibition or visit. Where experience of the app was seamless visitors reported an enhanced experience of Kew as needs, expectations and motives for visiting were met. The corollary was that where the app was not seamless visitors reported frustration and turned to old technology instead. Consequently, for digital mobile apps to become a successful part of the visitor experience these need to be designed and developed to provide a seamless experience that is in accordance both with the visitor's expectation of technology and with their individual motivation for visiting.

\section{REFERENCES}

Hooper-Greenhill, E. (1994) Museums and their visitors, Routledge, London and New York.

Kew Royal Botanic Gardens (2012). http://www.kew.org/visit-kew-gardens/visitinformation/mobile-app/ (retrieved 11 April 2012) .

Kew Royal Botanic Gardens http://www.kew.org/ (retrieved 3 April 2012).

Saunders, M., and Waterson, N. (2012) Delightfully lost: a new kind of wayfinding at kew. Museums and the Web, San Diego, USA, 11-14 April 2012. 
Susie Fisher Group (2011) Kew app evaluation: objectives and discussion guide (Unpublished report).

Susie Fisher Group (2011: 2) What is the visitor experience of using the new kew mobile app? Qualitative evaluation (Unpublished report).

Weiser M. and Brown, J. (1996) The coming age of calm technology. Xerox PARC, October 5, 1996. www.johnseelybrown.com/calmtech.pdf (retrieved 30 May 2012).
Wikipedia.

Augmented

reality http://en.wikipedia.org/wiki/Augmented_reality (retrieved 11 April 2012)

Wikipedia:

$Q R$

code. http://en.wikipedia.org/wiki/QR_code (retrieved 11 April 2012). 